\title{
Predicting outcomes and improving care in children with congenital kidney anomalies
}

\author{
Douglas G. Matsell ${ }^{1}$ (D) Marisa Catapang ${ }^{1}$ \\ Received: 14 June 2020 / Accepted: 16 June 2020 / Published online: 8 July 2020 \\ (C) IPNA 2020
}

Keywords Children · CAKUT · Congenital urinary tract obstruction · Obstructive nephropathy $\cdot$ Chronic kidney disease Biomarkers · Outcome

\section{Introduction}

Children born with congenital anomalies of the kidney and urinary tract (CAKUT) are at risk of progressive chronic kidney disease (CKD) and long-term kidney injury $[1,2]$. These observations are supported by long-term observational data; however, reports are limited and some are hampered by their retrospective nature, small cohort sizes, short follow ups, and heterogeneous patient populations [3]. Although a variety of CAKUT conditions are thought to have universally good long-term outcomes, such as children born with a solitary functioning kidney due to contralateral renal agenesis or a multicystic dysplastic kidney, a progressive decline in kidney function over time has been reported [4].

\section{Congenital urinary tract obstruction and obstructive nephropathy}

Congenital urinary tract obstruction (CUTO) resulting in obstructive nephropathy, the focus of the recent publication by McLeod et al. in this edition of Pediatric Nephrology [5], is singularly the most important and most common congenital kidney anomaly resulting in progressive CKD and kidney failure in young boys. Experimental models developed over the past 3 decades have defined the pathogenesis of the progressive and chronic kidney changes that occur, including the development of kidney dysplasia, medullary hypoplasia,

Douglas G. Matsell

dmatsell@cw.bc.ca

1 Division of Nephrology, British Columbia Children's Hospital, University of British Columbia, 4480 Oak Street, Room K4-150, Vancouver, British Columbia V6H 2V2, Canada interruption in nephron induction with a decrease in kidney size and nephron number, tubulointerstitial changes, and remodeling of the collecting duct epithelium [6]. The onset of obstructive kidney injury starts during fetal life, with the extent and scope of the pathological changes determined by the severity and duration of the obstruction. The causes are most commonly due to bladder outlet obstruction and posterior urethral valves (PUV) but include unilateral and bilateral functional and anatomical blockage of the ureters. Consequently, not all children are equally affected by the obstruction, some have associated anomalies or genetic conditions affecting multiple organ systems, and others have complicated antenatal and/or postnatal courses which negatively impact their longterm outcome [7]. Because of the variability of fetal kidney injury, there is a corresponding variability in the long-term postnatal clinical outcomes, including the development of hypertension and proteinuria, the extent of decline in kidney function, and the rate of progression to kidney failure. Previous investigations have focused on correlating the histopathological and functional changes seen in early experimental and human models of fetal obstruction to postnatal kidney function and longer-term clinical outcome, thereby providing clues to the pathogenesis of progressive loss of kidney function over time in this condition [8].

\section{Candidate biomarkers of disease progression in CUTO}

Obstructing urinary flow in a developing fetal kidney results in developmental kidney injury [9]. There are specific reproducible changes in the developing kidney that occur in the various kidney compartments which are conserved across mammalian species. Many of the histopathological changes, particularly those involved in and located at the epithelial 
urinary interface, are reflected in the urinary excretion patterns. Urine samples can be obtained from fetal to early postnatal life. Antenatally, these can be obtained somewhat invasively by needle aspiration from the fetal bladder or from amniotic fluid; postnatally, they can be collected noninvasively from routine urine samples. Proteins shown to be altered in human and animal models of CUTO, and thought to play a role in the pathogenesis of progressive kidney injury, are reflected in the urinary profiles of boys with PUV at various ages and differing stages of CKD. More severe kidney injury is reflected by larger changes in measured biomarkers, such as transforming growth factor- $\beta 1$ (TGFB) and L1 cell adhesion molecule (L1CAM) recovered from whole urine and urinary exosomes [10]. The value of this type of "biased" approach is that it identifies a protein or proteins known to be disrupted in experimental congenital kidney obstruction and whose role in the pathogenesis of the pathological changes is confirmed through yet other experimental models [11]. However, the challenges of using candidate proteins as biomarkers of outcome in CUTO include detecting and measuring the protein non-invasively in amniotic fluid or urine, and then showing that the excretion of the marker correlates with postnatal kidney function and longer-term clinical outcome. To achieve this, the postnatal kidney outcomes need to be clearly defined and standardized across studies to enable comparison. In addition, the biomarker should be a robust candidate protein, being implicated early in the process of injury, with disruption of the expression and function of the protein having a significant impact on long-term outcome. Examples include alteration in genes and proteins responsible for branching morphogenesis and nephron number determination, or genes and proteins responsible for fetal tubulointerstitial injury $[12,13]$. Unfortunately, the ability of a single candidate biomarker to predict outcome in CUTO may be limited. As highlighted recently by Murugapoopathy and colleagues, only approximately $20 \%$ of all children studied with CAKUT have an identifiable monogenic cause, and the majority of cases are likely explained by multigenetic, epigenetic, and environmental factors [14].

Other approaches have used high throughput strategies. Examples include mass spectrometry analyzing the urinary peptidome of fetuses with PUV, where a number of peptides, including collagen isoforms, had high predictive value for postnatal kidney outcomes [15]. Candidate proteins have also been captured by antibody array and quantified with enzymelinked immunosorbent assay from the urine of preterm infants immediately after birth, and at 1 year of postnatal life [16].

The utility of a new biomarker also lies in its ability to outperform conventional biomarkers in predicting the clinical outcome of interest. Clinical biomarkers such as fetal urine analytes and postnatal nadir serum creatinine, glomerular filtration rate (GFR) at 1 year of age, and proteinuria have been used to identify children with CUTO who may be at risk for the development of kidney failure [7, 17, 18], while other characteristics such as pulmonary hypoplasia and kidney dysplasia have been associated with mortality in this patient population [19]. To be clinically useful, new biomarkers (either candidate proteins or those discovered through unbiased omic approaches) should perform equally as well as previously described clinical biomarkers and/or be used to improve their performance.

\section{Prediction of outcome and risk stratification}

Biomarker discovery can serve to generate new knowledge about the pathogenesis of disease progression and, as highlighted by the work of McLeod and others in this issue of Pediatric Nephrology, to identify those children at risk of this progression. Biomarkers can be used either alone or in combination with other biomarkers to predict outcome. However, the association of the expression of a single biomarker with an outcome does not necessarily mean it predicts that outcome. Using a single clinical biomarker such as estimated GFR at 1 year of age to accurately predict the long-term outcome of kidney failure in boys with CUTO requires a large sample size, a significant number of cases reaching the outcome, and long and standardized follow up to mitigate bias. Rather, predictive models using multiple variables shown to correlate with outcome can be used to generate risk scores for that outcome. Not only do these multivariate models offer the possibility of identifying the most important new or previously identified variables that relate to a particular outcome, they are also essential to stratifying risk and allocating effort and resources according to that risk [20]. Practice patterns that may benefit from risk-based standardized care include the length of long-term follow up, frequency of clinic visits and assessments, types of assessment, ongoing laboratory testing and surveillance including imaging, discharge from clinic, and timing of transition to adulthood. Children at higher risk of developing long-term complications will likely require more intensive follow-up, while those with predicted excellent long-term outcomes may be appropriately followed up by primary care providers with minimal investigation and intervention.

Unfortunately, there has been a paucity of clinical guidelines for the care of children with congenital anomalies of the kidney including those with CUTO and obstructive uropathy. Some groups have correlated specific clinical characteristics in their local cohorts with worse outcomes. These observations have led to proposed care plans for these children. In children with a solitary functioning kidney (SFK), for example, Westland has identified CAKUT and lack of compensatory kidney growth in the SFK as risk factors for long-term kidney injury [21] and accordingly made recommendations for long-term care based on these observations. These and 
Fig. 1 CAKUT clinical pathway development model. A schematic representation of a proposed plan for developing a local CAKUT clinical pathway. Four key developmental phases are anticipated, each incorporating various key activities, as described. As new evidence emerges and changes to the predictive model and/or clinical pathway are required, the cycle may "loop back" on itself for major (solid arrow) and minor (dashed arrow) revisions, thereby supporting continuous improvement

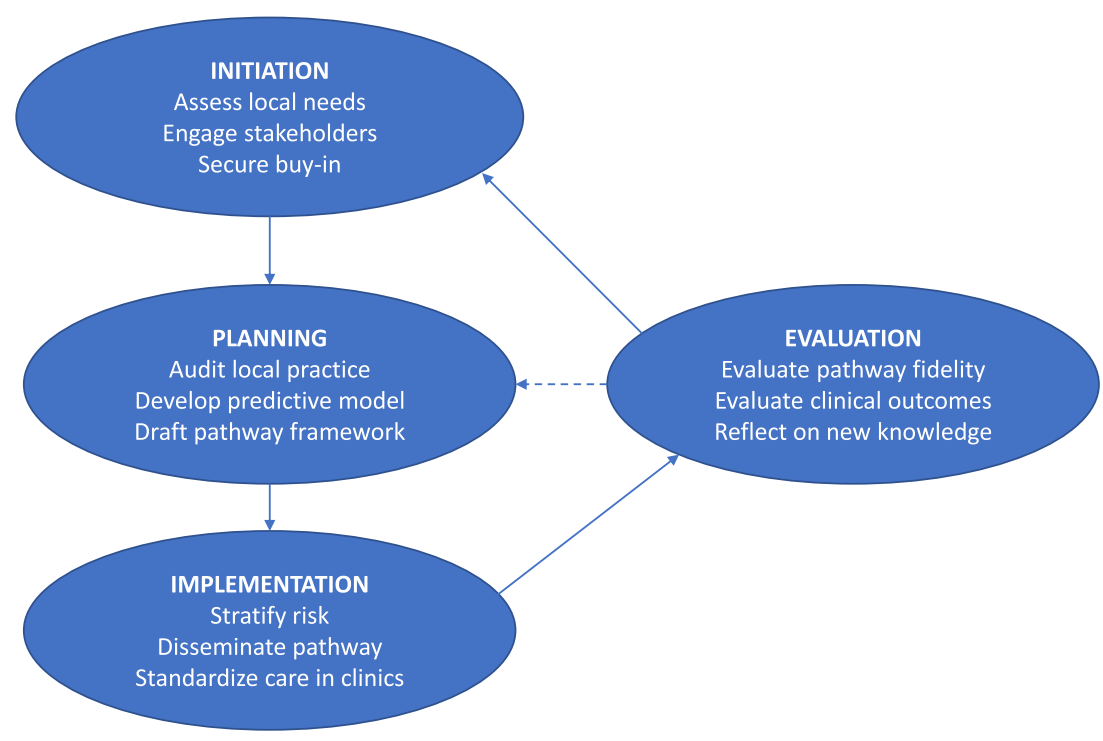

other published recommendations for follow-up schedules and investigations in children with SFK are important first steps to address the issue of standardizing care and stratifying risk in children with this relatively common childhood kidney disorder; however, they are not predictive models with risk scores, but more opinion-based recommendations which are influenced by regional practice biases and variations [22-24]. Until recently, similar recommendations for standardized care for children with CUTO and obstructive uropathy have been lacking.

Predictive models that use robust long-term data and incorporate multiple relevant biomarkers are essential for developing a standardized clinical pathway for care. To date, predicting outcome in children with CUTO and obstructive uropathy has focused on associating one or more conventional clinical biomarkers to the development of CKD and/or kidney failure. Most recently, Vasconcelos and colleagues have identified baseline GFR, nadir GFR, and proteinuria as predictors for the outcome of CKD in this population; they have developed a risk score based on the strengths of these associations, and they have shown good internal validation of the risk score [25]. The potential usefulness of this work is that it could be translated into improvements in clinical care-for discussions with parents regarding prognosis and ultimately to stratify a patient's risk of developing the CKD outcome and standardizing care accordingly. This would enable timely intervention in high-risk patients when needed, and for those patients who are at low risk, potentially minimizing unnecessary testing, and reducing the number of outpatient clinic visits.

The challenge of this work, and of most of the work analyzing long-term outcomes in children with obstructive uropathy and with other specific congenital kidney anomalies, is that much of the data collection is done retrospectively through historical center and practice records [26]. Consequently, the analyses are limited by missing data, the need for data imputation, and are restricted to conventional biomarkers. Furthermore, while the models may be locally applicable, to be universally adaptable to the clinical care of all children with CUTO, they require external validation, by adapting and analyzing the performance of similar datasets from other centers.

\section{Towards developing a clinical pathway}

Developing predictive models and standardized clinical pathways requires dedicated effort to define, collect, analyze, and validate clinical data from local cohorts of patients. Nevertheless, this is important work. Predictive models lay the groundwork for developing a clinical pathway framework. The most robust frameworks are evidence-based (thus, the predictive models use local data and practice audit), satisfy a perceived local need with major stakeholders identified (buyin), include a strategy for implementation, and enable ongoing analysis and change to incorporate new data or advancements in the field (Fig. 1). This is where work like that of McLeod and colleagues comes in [5]. Although there are limitations of their report given its retrospective nature, the heterogeneous population of children studied, the enrollment of patients later in the course of their illness, and the bias in selection of biomarkers, it does however demonstrate the potential for novel biomarkers to be incorporated into predictive models generated locally. This can be done going forward, built upon a framework of standardized care for the child with CUTO in the clinic. If, for example, the care of a child with CUTO and obstructive uropathy deemed to be at higher risk of CKD progression requires a visit to the clinic every 3 months with an appropriately measured blood pressure, urine protein estimation, and estimated GFR, then this is also an opportunity to prospectively collect biosamples at the point of care, for the 
measurement of previously described non-conventional biomarkers and of those novel biomarkers yet to be described.

This platform will enable a "looping back," a quality assurance cycle of sorts, to investigate how these novel biomarkers can substantially affect the risk stratification of children with congenital kidney anomalies. Taken together, well-informed predictive modeling and subsequent clinical pathway development can enable improved and more efficient care of this patient population.

\section{References}

1. Wong CJ, Moxey-Mims M, Jerry-Fluker J, Warady BA, Furth SL (2012) CKiD (CKD in children) prospective cohort study: a review of current findings. Am J Kidney Dis 60:1002-1011

2. Sanna-Cherchi S, Ravani P, Corbani V, Parodi S, Haupt R, Piaggio G, Innocenti ML, Somenzi D, Trivelli A, Caridi G, Izzi C, Scolari F, Mattioli G, Allegri L, Ghiggeri GM (2009) Renal outcome in patients with congenital anomalies of the kidney and urinary tract. Kidney Int 76:528-533

3. Wuhl E, van Stralen KJ, Verrina E, Bjerre A, Wanner C, Heaf JG, Zurriaga O, Hoitsma A, Niaudet P, Palsson R, Ravani P, Jager KJ, Schaefer F (2013) Timing and outcome of renal replacement therapy in patients with congenital malformations of the kidney and urinary tract. Clin J Am Soc Nephrol 8:67-74

4. Westland R, Schreuder MF, Bokenkamp A, Spreeuwenberg MD, van Wijk JA (2011) Renal injury in children with a solitary functioning kidney-the KIMONO study. Nephrol Dial Transplant 26: 1533-1541

5. McLeod DJ, Sebastiao YV, Ching CB, Greenberg JH, Furth SL, Becknell B (2020) Longitudinal kidney injury biomarker trajectories in children with obstructive uropathy. Pediatr Nephrol https:// doi.org/xxxxxxxxxxxxxxxxxxxxxxxxxxxxxxxxxxxxxx

6. Matsell DG, Tarantal AF (2002) Experimental models of fetal obstructive nephropathy. Pediatr Nephrol 17:470-476

7. Matsell DG, Yu S, Morrison SJ (2016) Antenatal determinants of long-term kidney outcome in boys with posterior urethral valves. Fetal Diagn Ther 39:214-221

8. Chevalier RL (2016) Prognostic factors and biomarkers of congenital obstructive nephropathy. Pediatr Nephrol 31:1411-1420

9. Trnka P, Hiatt MJ, Tarantal AF, Matsell DG (2012) Congenital urinary tract obstruction: defining markers of developmental kidney injury. Pediatr Res 72:446-454

10. Trnka P, Ivanova L, Hiatt MJ, Matsell DG (2012) Urinary biomarkers in obstructive nephropathy. Clin J Am Soc Nephrol 7: $1567-1575$

11. Ivanova L, Butt MJ, Matsell DG (2008) Mesenchymal transition in kidney collecting duct epithelial cells. Am J Physiol Renal Physiol 294:F1238-F1248

12. Gewin L, Bulus N, Mernaugh G, Moeckel G, Harris RC, Moses HL, Pozzi A, Zent R (2010) TGF-beta receptor deletion in the renal collecting system exacerbates fibrosis. J Am Soc Nephrol 21:1334 1343
13. Deng H, Zhang Y, Xiao H, Yao Y, Liu X, Su B, Zhang H, Xu K, Wang S, Wang F, Ding J (2019) Diverse phenotypes in children with PAX2-related disorder. Mol Genet Genomic Med 7:e701

14. Murugapoopathy V, Gupta IR (2020) A primer on congenital anomalies of the kidneys and urinary tracts (CAKUT). Clin J Am Soc Nephrol 15:723-731

15. Buffin-Meyer B, Tkaczyk M, Stanczyk M, Breuil B, Siwy J, Szaflik K, Talar T, Wojtera J, Krzeszowski W, Decramer S, Klein J, Schanstra JP (2020) A single-center study to evaluate the efficacy of a fetal urine peptide signature predicting postnatal renal outcome in fetuses with posterior urethral valves. Pediatr Nephrol 35:469 475

16. Charlton JR, Norwood VF, Kiley SC, Gurka MJ, Chevalier RL (2012) Evolution of the urinary proteome during human renal development and maturation: variations with gestational and postnatal age. Pediatr Res 72:179-185

17. Sarhan OM, El-Ghoneimi AA, Helmy TE, Dawaba MS, Ghali AM, el Ibrahiem HI (2011) Posterior urethral valves: multivariate analysis of factors affecting the final renal outcome. J Urol 185:24912495

18. McLeod DJ, Ching CB, Sebastiao YV, Greenberg JH, Furth SL, McHugh KM, Becknell B (2019) Common clinical markers predict end-stage renal disease in children with obstructive uropathy. Pediatr Nephrol 34:443-448

19. Herbst KW, Tomlinson P, Lockwood G, Mosha MH, Wang Z, D'Alessandri-Silva C (2019) Survival and kidney outcomes of children with an early diagnosis of posterior urethral valves. Clin J Am Soc Nephrol 14:1572-1580

20. Au EH, Francis A, Bernier-Jean A, Teixeira-Pinto A (2020) Prediction modeling-part 1: regression modeling. Kidney Int 97: 877-884

21. Westland R, Kurvers RA, van Wijk JA, Schreuder MF (2013) Risk factors for renal injury in children with a solitary functioning kidney. Pediatrics 131:e478-e485

22. Westland R, Schreuder MF, van Goudoever JB, Sanna-Cherchi S, van Wijk JA (2014) Clinical implications of the solitary functioning kidney. Clin J Am Soc Nephrol 9:978-986

23. Corbani V, Ghiggeri GM, Sanna-Cherchi S (2011) Congenital solitary functioning kidneys: which ones warrant follow-up into adult life? Nephrol Dial Transplant 26:1458-1460

24. Urisarri A, Gil M, Mandia N, Aldamiz-Echevarria L, Iria R, Gonzalez-Lamuno D, Couce ML (2018) Retrospective study to identify risk factors for chronic kidney disease in children with congenital solitary functioning kidney detected by neonatal renal ultrasound screening. Medicine 97:e11819

25. Vasconcelos MA, ACS ES, Gomes IR, Carvalho RA, Pinheiro SV, Colosimo EA, Yorgin P, Mak RH, Oliveira EA (2019) A clinical predictive model of chronic kidney disease in children with posterior urethral valves. Pediatr Nephrol 34:283-294

26. Poggiali IV, Simoes ESAC, Vasconcelos MA, Dias CS, Gomes IR, Carvalho RA, Oliveira MCL, Pinheiro SV, Mak RH, Oliveira EA (2019) A clinical predictive model of renal injury in children with congenital solitary functioning kidney. Pediatr Nephrol 34:465474

Publisher's note Springer Nature remains neutral with regard to jurisdictional claims in published maps and institutional affiliations. 